\title{
Repetition effects in directed forgetting: evidence for retrieval inhibition
}

\author{
R. EDWARD GEISELMAN and BEHROOZ BAGHERI \\ University of California, Los Angeles, California
}

\begin{abstract}
Four experiments were conducted in support of a role for memory retrieval inhibition in directed forgetting. In each experiment, subjects were presented a list of words, some of which they were instructed to remember and some of which they were instructed to forget. After a recall test for all the words, the list was repeated. This time, however, all the words were presented with instructions that they be remembered. The improvement in recall from Trial 1 to Trial 2 was greater for the "forget" (F) words than for the "remember" (R) words. This difference was not due to a memorization-difficulty, item-selection effect (Experiment 2), a differential priority for rehearsal or output position given to the F items on Trial 2 (Experiment 3), or the greater number of $\mathrm{F}$ items left to be learned after Trial 1 (Experiment 4). Thus, the differential improvement from List 1 to List 2 for the $F$ items was interpreted as a release of retrieval inhibition owing to the change in cue from forget to remember.
\end{abstract}

A considerable amount of interest has been generated throughout psychology toward understanding mechanisms of motivated forgetting. That interest can be seen in studies and writings on repression, suppression, and certain forms of amnesia. One popular method for studying mechanisms of intentional forgetting in the laboratory involves instructing subjects to forget certain items presented during the course of an experiment.

In one variant of the instructed-forgetting paradigm, subjects are given either a "forget" command or a "memorize" command immediately following the presentation of each item in a list, with the two types of commands interleaved randomly throughout the list. Upon completing the list, the subjects are told to recall all the items that have been presented, sometimes including the items that they previously had been told to forget. With this procedure, recall of the to-be-forgotten $(F)$ items has been observed to be reliably lower than recall of the tobe-remembered $(R)$ items. If not given until just before the time of test, however, the forget command typically has little, if any, effect on the recall of the F items (Bjork, 1970; Roediger \& Tulving, 1979). Thus, the principal focus of these studies traditionally has been on mechanisms that are operative at encoding, such as selective rehearsal and selective grouping (Bjork, 1972; Geiselman, 1974). Several alternative explanations of the results have been largely discounted. These include erasure (Bjork, 1972), response suppression (Weiner, 1968), output interference (Geiselman, Bjork, \& Fishman, 1983), and disrupted memory trace consolidation (Bjork \& Geiselman, 1978). However, Weiner and Reed (1969) found that commands to forget led to greater forgetting of target items

The authors' mailing address is: Department of Psychology. University of California, Los Angeles, California 90024. than did commands instructing subjects simply not to rehearse the items. This result is consistent with the hypothesis that intentional forgetting does not simply terminate rehearsal, but, rather, can lead to an inhibition of memories.

More recently, Geiselman, Bjork, and Fishman (1983) presented a series of experiments that supported a role for retrieval mechanisms in intentional forgetting. In those experiments, subjects were presented a list of words in which every other word was to be remembered and the remaining words were to be judged on pleasantness, but were not to be remembered. After half of the entire list had been presented, the subjects were told that what they had done thus far had been practice and that they should forget about what they had memorized to that point. It was found that the command to forget negatively affected later memory for the to-be-judged items, which the subjects had no intent to remember, as well as memory for the items learned intentionally. This result, which was found in recall but not in recognition, is difficult to explain in terms of encoding mechanisms. Geiselman, Bjork, and Fishman (1983) argued that, when it was presented, the command to forget initiated a process that served to inhibit or block access routes to the list items in episodic memory.

Geiselman, Bjork, and Fishman (1983) also noted several parallels between the nature of the recall protocols in studies of directed forgetting and studies of hypnotically induced amnesia. Disrupted retrieval has been implicated with the hypnosis paradigm because the forget instruction that affects memory recall is given just before the memory test and because a later countermand reversing the forget instruction serves to enhance memory recall. With or without hypnosis, to-be-forgotten items that can be recalled are typically recalled in an order different from that in which they were input. 
Additional evidence for retrieval inhibition was found in an experiment by Geiselman, MacKinnon, Fishman, Jaenicke, Larner, Schoenberg, and Swartz (1983) on individual differences in forgetting. In that study, subjects participated in both a standard posthypnotic amnesia procedure, in which retrieval inhibition has been implicated (Kihlstrom, 1977), and a nonhypnotic intentionalforgetting procedure. In the hypnosis procedure, a suggestion was given, just before recall, to forget the previous events that had taken place in the experiment; also given was a countermand to signal the removal of the suggestion at a later time. Subjects who exhibited the greatest retrieval inhibition with hypnosis prior to the countermand showed the lowest F-item recall in a nonhypnotic experiment; and, independently, the subjects who showed the greatest inhibition release following the countermand showed the highest $\mathrm{F}$-item recall. These findings indicate that the forgetting processes in both paradigms are related, and the implication is that some of the mechanisms are the same. Because encoding deficits can be ruled out in the hypnosis paradigm, retrieval inhibition is implicated in the nonhypnotic case. Independent evidence for disrupted retrieval again was found in analyses of the recall orders.

The present experiments were designed to seek, using an item-repetition paradigm, further evidence for a mechanism of memory retrieval inhibition without hypnosis. It was hypothesized that the recall of $F$ items differs, in part, from the recall of $R$ items because the $F$ items suffer from retrieval inhibition. Therefore, a re-presentation of unrecalled $F$ items, presented now as $R$ items, should have a powerful effect on the recall of those items relative to the effect of a re-presentation of unrecalled $R$ items as $R$ items. This outcome is predicted because initially unrecalled $R$ items would be affected only by the second study opportunity, whereas the initially unrecalled $\mathrm{F}$ items would be positively affected by a release of the inhibition, as a result of the change in cue from forget to remember, plus the second study opportunity.

Some evidence in support of this prediction has been reported by Reed (1970) and Weiner and Reed (1969, Experiment 3 ). In the Weiner and Reed experiment, subjects were instructed on each Brown-Peterson trial to either forget or rehearse a letter trigram. Each trigram appeared twice in the sequence of trials. Of particular interest is a comparison of recall performance for items that were cued $F$ on the first presentation but $R$ on the second presentation with items that were cued $R$ on both presentations. Even though recall performance on the first presentation was characteristically lower for the $\mathrm{F}$ items than for the $\mathrm{R}$ items, recall performance on the second presentation was the same regardless of the cue on the first presentation. Thus, Weiner and Reed concluded that forget commands must have some influence on memory retrieval rather than solely on memory storage. Otherwise, recall performance for the $F$ items should not have recovered completely when re-presented as $\mathrm{R}$ items.
The present experiments were designed to replicate and extend the work of Weiner and Reed (1969). In particular, their finding of equivalent recall for $R$ and $F$ items following repetition could be explained as enhanced encoding of the $\mathrm{F}$ items at repetition, thus compensating for the initial encoding deficit resulting from the $F$ cues. This argument was made by Bjork (1972, p. 228) and by Geiselman, Bjork, and Fishman (1983, pp. 61-62). Thus, in the present Experiment 1, the comparison of repetition effects on the recall of $R$ versus $F$ items was restricted to only those items that were not recalled on the first presentation but were recognized on the second presentation as having been presented before. This analysis was carried out to equate the $R$ and $F$ items on initial encoding and storage such that a greater improvement in recall for the $F$ items following repetition could be attributed to retrieval factors, specifically to a release of the initial retrieval inhibition. Alternative explanations of the results that were obtained in Experiment 1 were tested in Experiments 2,3 , and 4 .

\section{EXPERIMENT 1}

In Experiment 1, the subjects first were presented a list of words with some items cued as to-be-remembered (R) items and some cued as to-be-forgotten (F) items. Subsequent memory was tested for all items. Then, on a second trial, some $F$ items and $R$ items were repeated, but this time all words were presented as $R$ items. Trial 2 memory for $\mathrm{R}$ and $\mathrm{F}$ items not recalled on Trial 1 was examined using both a recognition and a recall procedure.

\section{Method}

Subjects. The subjects were 21 undergraduate volunteers from the introductory psychology course at the University of California, Los Angeles. The subjects were tested in groups of 4 to 7 .

Materials and Procedure. In the first part of the experiment, a list of 36 four-letter nouns was presented to the subjects visually on slides. Each word slide was presented for $5 \mathrm{sec}$ and was followed by either a "forget" slide or a "blank" slide for $5 \mathrm{sec}$. The order of presentation of the instruction slides was mixed throughout the list with the restriction that no more than two instances of either type appear in sequence. The subjects were told that a blank slide meant that they should try to memorize the word that immediately preceded it because they would be asked to recall it later. A forget slide meant that they should forget the word that immediately preceded it. The $\mathrm{R}$ words and $\mathrm{F}$ words were counterbalanced across subjects.

After the list had been presented, the subjects solved mathematical deductive-reasoning problems for $3 \mathrm{~min}$. Then they were told to write down any word that they could remember from the experiment, including the forget words. The recall period was $7 \mathrm{~min}$. The first two and last two words in the list served as buffer items and were not scored. The subjects were told, "Although you were allowed to forget the words that were followed by a forget slide, I now want you to write down any of those words that you can remember. It is important for the experiment that you recall all the forget words that you can possibly remember."

In the second part of the experiment, the subjects were presented a list of words that consisted mostly of the same words as before, but eight of the words (four $R$ words and four $F$ words) were 
replaced with new words. The words that were replaced were selected such that one $\mathrm{R}$ word and one $\mathrm{F}$ word were removed from each quarter of the list. The subjects were instructed to memorize all the words in this list, so only blank slides were used as instruction slides. Recognition of the words from List 1 was tested by requiring the subjects to judge whether or not each word had been presented in List 1 . These judgments were made by having the subjects circle YES or NO for each word during the presentation of List 2.

Upon completion of the list, the subjects first solved additional deductive-reasoning problems for $3 \mathrm{~min}$ and then recalled the words from the list for $7 \mathrm{~min}$. The first two and last two words in the list served as buffer items and were not scored.

\section{Results and Discussion}

The unconditional probabilities of word recall on Trial 1, recognition on Trial 2, and recall on Trial 2 are presented in Table 1 for the items cued as $R$ and $F$ items on Trial 1. As expected, recall for $F$ items on Trial 1 was poor even though the subjects were encouraged to write down all the items from the list. In contrast, recognition performance on Trial 2 for these items was substantial, although still inferior to recognition for the $R$ items $[F(1,20)=14.40, p<.001]$. Recall performance following Trial 2 was inferior for items initially cued as $F$ items $[F(1,20)=32.19, p<.001]$, but the difference was less pronounced than on Trial 1. The higher absolute level of recall on Trial 2 for $R$ items could be due to either greater selective rehearsal or greater retrieval practice for $\mathrm{R}$ items on Trial 1 .

Of principal importance here is the conditional probability of recall on Trial 2 given that an item was not recalled on Trial 1. It was hypothesized that the unrecalled $F$ items from Trial 1 would derive greater benefit from the re-presentation on Trial 2 than would unrecalled $R$ items. This is because the unrecalled $F$ items would not only receive a second opportunity for rehearsal, as would the unrecalled $\mathrm{R}$ items, but would also show an enhancement due to a release of the retrieval inhibition that was invoked on Trial 1. The results were consistent with this prediction. The probability of recall on Trial 2 for items not recalled on Trial 1 was .45 for the $F$ items but only .28 for the $R$ items $[F(1,20)=6.77, p<.02]$.

Since recognition during Trial 2 was marginally lower for the $\mathrm{F}$ items than for the $\mathrm{R}$ items, it was possible that the subjects devoted more rehearsal time to the $F$ items during their study of List 2 . This would be the case if subjects decided to study well those items that they had not yet learned. Therefore, to restrict the analysis to items that the subjects recognized on Trial 2, the conditional probability of recall on Trial 2 was computed only for

Table 1

Memory Performance in Experiment 1

\begin{tabular}{lccccc}
\hline & \multicolumn{3}{c}{ Trial 1 Trial 2 } \\
Type & $\begin{array}{c}\mathrm{Recall} \\
\left(\mathrm{Rc}_{2}\right)\end{array}$ & $\begin{array}{c}\mathrm{Recall} \\
\left(\mathrm{Rc}_{2}\right)\end{array}$ & $\left(\mathrm{Rc}_{2} \mid \overline{\mathrm{Rc}_{1}}\right)$ & $\begin{array}{c}\text { Trial 2 } \\
\mathrm{Recognition} \\
\left(\mathrm{Ro}_{2}\right)\end{array}$ & $\left(\mathrm{Rc}_{2} \mid \overline{\mathrm{Re}_{1}} \cap \mathrm{Ro}_{2}\right)$ \\
\hline Remember & .58 & .65 & .28 & .86 & .31 \\
Forget & .10 & .49 & .45 & .76 & .47 \\
\hline
\end{tabular}

items that were not recalled on Trial 1 but were recognized on Trial 2. The results of this analysis again were consistent with the retrieval inhibition hypothesis: The conditional probability was .47 for $F$ items versus .31 for $\mathrm{R}$ items $[\mathrm{F}(1,20)=4.47, \mathrm{p}<.05]$. The possibility of a priority for rehearsal of the $F$ items was tested further in Experiment 3 using a between-subjects design on Trial 2.

The results of Experiment 1 are in agreement with the theory that retrieval inhibition plays a significant role in intentional forgetting. An alternative explanation of the data can be offered, however, in terms of a word-selection effect. Given that, on Trial 1, the subjects were trying to memorize the $\mathrm{R}$ words but not the $\mathrm{F}$ words, perhaps the unrecalled $\mathrm{R}$ words were more difficult than many of the unrecalled $\mathrm{F}$ words. If so, then it is not surprising that the re-presentation of the unrecalled $F$ items led to a greater enhancement of performance than the representation of the unrecalled $R$ items. Even though all the words were common four-letter nouns, they could have varied on imagery value, ease of grouping, and so forth.

To examine the possibility of item selection, one additional group of 21 subjects served as yoked controls in a one-trial free-recall task. In this condition, each subject received the unrecalled $F$ items and $R$ items of one of the original subjects. These lists ranged in length from 10 to 22 words. The order of presentation for the words was preserved, with a 5 -sec rate of presentation, no $\mathrm{F}$ cues, a 3-min distraction task, and a 7-min recall period. No evidence for differential item difficulty was found: The proportion of yoked $R$ items recalled was .42 , and the proportion of yoked $F$ items recalled was $.40(t<1)$. Although this result is useful information, it does not rule out the possibility of a subject $\times$ item difficulty interaction. Therefore, Experiment 2 was conducted to provide a further test of the item-difficulty selection hypothesis.

\section{EXPERIMENT 2}

Given the possibility of an item-selection effect in Experiment 1 , the ideal comparison for the present purposes would be between Trial 2 recall for unrecalled $F$ items and Trial 2 recall for unrecalled items that the subjects were neither trying to memorize nor trying to forget on Trial 1. Such a comparison would allow for an evaluation of inhibitory effects of $F$ cues without item-selection effects due to the difficulty of word memorization on Trial 1. Therefore, in Experiment 2, subjects were presented with three types of words on Trial 1: $\mathrm{R}$ words, $F$ words, and words that were neither to be remembered nor to be forgotten, but rather were to be judged on pleasantness (J words).

The retrieval-inhibition theory predicts that the improvement in recall on Trial 2 for unrecalled $F$ items should be greater than the improvement in recall on Trial 2 for either the unrecalled $R$ items or the unrecalled $\mathrm{J}$ items. This is because only the unrecalled $\mathrm{F}$ items can 
benefit from a release of retrieval inhibition on Trial 2 . The item-difficulty selection hypothesis predicts that the improvement in recall on Trial 2 for unrecalled $J$ items should be greater than the improvement for unrecalled $\mathrm{R}$ items, but should be comparable to the improvement in recall for unrecalled $F$ items. This is because the subjects are not attempting to memorize either the $J$ words or the $\mathrm{F}$ words on Trial 1 , and therefore effects of memorization difficulty would be a factor only in the Trial 2 recall of unrecalled $R$ items.

\section{Method}

Subjects. The subjects were 28 undergraduate volunteers from the introductory psychology course at the University of California, Los Angeles. The subjects were tested in groups of 4 to 7 .

Procedure. In the first part of the experiment, a list of 40 four-letter nouns was presented visually to the subjects. Each word slide was presented for $5 \mathrm{sec}$ and was followed for $5 \mathrm{sec}$ by either a "forget" slide, a "recall" slide, or a blank slide. The order of presentation of the instruction slides was mixed throughout the list, with the restriction that no more than two instances of one type appear in sequence. The subjects were told that a forget slide meant that they should forget the word that immediately preceded it. A recall slide meant that they should try to memorize the word because they would be asked to recall it later in the experiment. A blank slide meant that they should neither forget nor memorize the word, but rather that they should judge the word for pleasantness on a scale of 1 to 5 .

Following presentation of the list, the subjects solved mathematical deductive-reasoning problems for $3 \mathrm{~min}$. Then they were told to write down any word that they could remember from the experiment, including the forget words and judge words. Seven minutes was allowed for recall. The first two and last two words in the list served as buffer items and were not scored.

In the second part of the experiment, the subjects were presented the same list of words as before, with blank slides used as instruction slides throughout the list. As in Experiment 1, blank slides in the second part of the experiment meant "recall"; thus, all words were to be memorized. After solving mathematical problems for $3 \mathrm{~min}$, the subjects were asked to recall and write down all the words from the list in any order. The first two and last two words in the list served as buffer items and were not scored.

\section{Results and Discussion}

The unconditional probabilities of recall on Trial 1 and Trial 2, as well as the conditional probability of recall on Trial 2 given nonrecall on Trial 1, are presented in Table 2 for the $\mathrm{R}, \mathrm{J}$, and $\mathrm{F}$ items. It is evident from Table 2 that the enhancement of memory recall due to the representation of unrecalled J words (.34) is much like the enhancement of memory recall due to the re-presentation of unrecalled $R$ words (.37). Given the greater enhancement for $F$ words (.46), these results provide further support for the retrieval-inhibition hypothesis. The main effect of cue type in the conditional-probability analysis was

Table 2

Memory Performance in Experiment 2

\begin{tabular}{|c|c|c|c|}
\hline $\begin{array}{l}\text { Word } \\
\text { Type }\end{array}$ & $\begin{array}{c}\text { Trial 1 } \\
\text { Recall } \\
\left(\mathrm{Rc}_{1}\right)\end{array}$ & $\begin{array}{l}\text { Trial } 2 \\
\text { Recall } \\
\left(\mathrm{Rc}_{2}\right)\end{array}$ & $\left(\mathrm{Rc}_{2} \mid \overline{\mathrm{R}_{1}}\right)$ \\
\hline Remember & .46 & .66 & .37 \\
\hline Judge & .32 & .55 & 34 \\
\hline Forget & .07 & .50 & .54 \\
\hline
\end{tabular}

significant $[\mathrm{F}(1,54)=20.09, \mathrm{MSe}=.02, \mathrm{p}<.001]$; and a Tukey's posttest showed that the memory recall improvement was significantly greater for the $F$ items than for either the $R$ or the $J$ items $(p<.01)$.

An argument might be offered that the judge task required a sufficient amount of processing such that the unrecalled $\mathrm{J}$ words from Trial 1 were selectively more difficult than the unrecalled $F$ words. This argument is not plausible, however, given that the $\mathrm{R}$ words received greater processing than the J words (as suggested by the significant difference in recall on Trial $1, \mathrm{p}<.05$ ) and yet the improvement in memory recall for the $\mathrm{R}$ words was the same as that for the $J$ words. Thus, there does not appear to have been an inverse correspondence between the extent of processing on Trial 1 and the amount of memory improvement on Trial 2 as would be predicted by the item-difficulty selection hypothesis. More generally, this outcome casts doubt on any alternative explanation of the conditionalized repetition results that is based upon differential storage strength of the items on Trial 1.

Although the pattern of results is contrary to the itemdifficulty selection hypothesis, the comparison between the orienting-task (judge) words and forget words would have been more direct had the level of Trial 1 recall for the orienting-task words been comparable to that for the forget words. Therefore, Experiment 2 was replicated with 18 new subjects whose orienting task on Trial 1 was to count the letters in the words. As anticipated, the results showed that this graphemic task produced poor recall on Trial 1 (.14). Trial 1 recall for $F$ items was comparable (.09), but the conditional probability of Trial 2 recall given nonrecall on Trial 1 was again greater for $F$ items (.49) than for the orienting-task items $(.30)[\mathrm{t}(17)=2.53$, $p<.05]$. Thus, the low level of Trial 1 recall for $F$ items is not responsible for the conditionalized recall results on Trial 2.

\section{EXPERIMENT 3}

The third experiment was conducted to determine whether the advantage for unrecalled $\mathrm{F}$ items on Trial 2 could be attributed to the subjects' giving priority to those items for rehearsal during Trial 2 . This hypothesis was tested using a between-subjects design on Trial 2. Half of the subjects were presented only the $F$ items from Trial 1 on the second list, whereas the remaining subjects were presented only the $R$ items from Trial 1 on the second list. Thus, differential priority for rehearsal of the $F$ versus the $R$ items was not possible on Trial 2 of this experiment.

The between-subjects design also allowed for a test of the possibility that output interference on Trial 1 contributed to the pattern of Trial 2 results in Experiments 1 and 2. Although output interference has been discounted elsewhere in directed-forgetting research (Geiselman, Bjork, \& Fishman, 1983), some potentially recallable $F$ items in the present experiments may not have been recalled on Trial 1 because the subjects searched for them only 
Table 3

Memory Performance in Experiment 3

\begin{tabular}{lccc}
\hline $\begin{array}{l}\text { Word } \\
\text { Type }\end{array}$ & $\begin{array}{c}\text { Trial } 1 \\
\text { Recall } \\
\left(\mathrm{Rc}_{1}\right)\end{array}$ & $\begin{array}{c}\text { Trial } 2 \\
\text { Recall } \\
\left(\mathrm{Rc}_{2}\right)\end{array}$ & $\left(\mathrm{Rc}_{2} \mid \mathrm{Rc}_{1}\right)$ \\
\hline Remember & .44 & .60 & .34 \\
Forget & .13 & .54 & .51 \\
\hline
\end{tabular}

after the $\mathrm{R}$ items had been recalled, not because the $\mathrm{F}$ items were inhibited by the F cues. Consequently, the subjects may have given those unrecalled but learned $F$ items differential priority for early output on Trial 2 . Such a scenario would predict an advantage for the unrecalled $F$ items in recall on Trial 2. This problem was circumvented in Experiment 3 for some subjects because the $R$ items were not presented for study or recall on Trial 2.

\section{Method}

Subjects. The subjects were 40 undergraduate volunteers from the introductory psychology course at the University of California, Los Angeles. All subjects participated in exchange for course credit. The subjects were tested in groups of 4 to 7 .

Materials and Procedure. The same list of 40 four-letter nouns that had been used in Experiment 2 was presented to all the subjects on Trial 1. A random half of the words were followed by a "forget" slide; the remaining words were followed by a blank ("remember") slide. The word slides and instruction slides were presented for $5 \mathrm{sec}$ each. Following completion of the 3-min interpolated task, the subjects were instructed to write down all the words that had been presented on the list. The first two and last two words from the list served as buffer items and were not scored.

On Trial 2, 20 of the subjects were shown the $18 \mathrm{~F}$ items from Trial 1, and 20 of the subjects were shown the $18 \mathrm{R}$ items from Trial 1. In each case, each of the items was followed by a blank slide and was presented as a to-be-remembered word.

\section{Results and Discussion}

The unconditional probability of recall on Trial 1 and the probability of recall on Trial 2 given nonrecall on Trial 1 are presented in Table 3 . As in the previous experiments, the improvement in recall was greater for the unrecalled $\mathrm{F}$ items than for the unrecalled $\mathrm{R}$ items, and this difference was significant $[\mathrm{t}(38)=2.36, \mathrm{p}<.01]$. Given the between-subjects design on Trial 2, the advantage for the unrecalled $\mathrm{F}$ items cannot be attributed to a priority for rehearsal or output position for $\mathrm{F}$ items over $\mathrm{R}$ items on Trial 2.

\section{EXPERIMENT 4}

One remaining explanation of the greater improvement in recall on Trial 2 for unrecalled $F$ items involves the greater number of $\mathrm{F}$ items that remained to be learned on Trial 2. In Experiment 3, all of the R or F items were repeated on Trial 2 for study. If it is assumed that the subjects reached a resource-limited upper bound on the total number of $\mathrm{R}$ or $\mathrm{F}$ words that they could process for recall on the two trials combined (e.g., 10 or 11 words in Experiment 3 ), then the proportion of unrecalled words learned on Trial 2 could have been artifactually greater for the $F$ items. This is because fewer $F$ items than $R$ items were learned on Trial 1 , such that the $\mathrm{R}$ items would have reached a resource-limited ceiling first. This hypothesis was tested in Experiment 4, with a between-subjects design on Trial 2, as in Experiment 3, but with exactly 10 unrecalled $\mathrm{R}$ items or 10 unrecalled $\mathrm{F}$ items being presented to each subject on Trial 2. Thus, Trial 2 recall was based on a fixed number of items to be learned such that a resource-limited ceiling would affect $\mathrm{R}$ - and F-item recall equally.

\section{Method}

Subjects. The subjects were 40 undergraduate volunteers from the introductory psychology course at the University of California, Los Angeles. The subjects participated in exchange for course credit and were tested in groups of 3 or 4 .

Materials and Procedure. The list of 40 words and the R and $F$ instruction slides from Experiment 3 were used as the materials. The presentation rate was increased to $3 \mathrm{sec}$ per slide. All subjects viewed the slides, completed the distraction task, and attempted to recall all the words.

Following Trial 1, all subjects were excused from the room for a 5 -min break, during which time the experimenter derived a list of 10 words for each subject individually, to be used on Trial 2 . For 20 of the subjects, the 10 words for Trial 2 were $R$ words that had not been recalled on Trial 1 . For the remaining 20 subjects, the 10 words were $F$ words that had not been recalled on Trial 1 . In all cases, the 10 words were selected randomly from the pool of $(\mathrm{R}$ or $\mathrm{F}$ ) words not recalled by that subject (excluding the primacy and recency buffer items). Two subjects in the $\mathrm{R}$-word condition were replaced because they recalled more than 8 (nonbuffer) $R$ words on Trial 1 . The 10 words for Trial 2 were presented to the subjects in the form of a list on a sheet of paper, and all the words were presented as to-be-remembered words. Each subject studied the personalized list for $30 \mathrm{sec}$, completed a distractor task for $3 \mathrm{~min}$, and then tried to recall the 10 words.

\section{Results and Discussion}

The recall results are presented in Table 4 . The conditionalized Trial 2 recall of $F$ items exceeded that of $R$ items $[t(38)=2.27, p<.05]$. Thus, equating the number of words to be learned on Trial 2 did not affect the advantage for $\mathrm{F}$ items, an outcome consistent with the retrieval-inhibition hypothesis.

\section{GENERAL DISCUSSION}

Much of the data gathered from nonhypnotic intentionalforgetting experiments can be explained by factors operative at encoding, but a strong case can also be made for a significant role of memory retrieval inhibition. Geisel-

Table 4

Memory Performance in Experiment 4

\begin{tabular}{|c|c|c|}
\hline $\begin{array}{l}\text { Word } \\
\text { Type }\end{array}$ & $\begin{array}{l}\text { Trial } 1 \\
\text { Recall }\end{array}$ & $\begin{array}{l}\text { Trial } 2 \\
\text { Recall* }\end{array}$ \\
\hline Remember & $\begin{array}{r}.29 \\
0.5\end{array}$ & $\begin{array}{l}.56 \\
70\end{array}$ \\
\hline
\end{tabular}

* Of 10 words not recalled on Trial 1. 
man, Bjork, and Fishman (1983) found that a forget cue can have a deleterious effect on items that subjects have no intent to remember in any case, and that the pattern of recall for $\mathrm{F}$ items appears to be disrupted. Furthermore, Geiselman, MacKinnon, et al. (1983) found a correspondence between individual differences in hypnotic amnesia (ascribed to retrieval inhibition) and individual differences in waking intentional forgetting. The present results complement the previous word in that unrecalled $\mathrm{F}$ items appear to be qualitatively different from unrecalled $R$ items. Specifically, a re-presentation of unrecalled $F$ items as $\mathrm{R}$ items resulted in a greater facilitation of memory performance than did a re-presentation of unrecalled $R$ items as $\mathrm{R}$ items. This difference was not due to a memorization-difficulty, item-selection effect (Experiment 2), a differential priority for rehearsal or output position given to the $F$ items on Trial 2 (Experiment 3), or the greater number of $F$ items left to be learned after Trial 1 (Experiment 4).

The sum pattern of results is consistent with the idea that $F$ items suffer from retrieval inhibition in addition to insufficient encoding. As proposed by Geiselman, Bjork, and Fishman (1983), it is hypothesized that a command to forget initiates a process that serves to inhibit or block access routes to the target items in episodic memory. Given such a mechanism, it is somewhat curious that a forget command has little effect on memory for items that receive elaborate precue processing (Bugelski, 1970) or on memory performance in general if the cue is given just before test (Bjork, 1970; Roediger \& Tulving, 1979). On the other hand, when hypnosis is used, a forget instruction is effective for inhibiting recall when items have been well learned (Kihlstrom, 1977) and when the instruction is presented just before test. Furthermore, a countermand often reverts recall performance to a high level (Geiselman, MacKinnon, et al., 1983; Kihlstrom \& Evans, 1976). It appears, therefore, that there are certain conditions in which retrieval inhibition can be manipulated near the time of item retrieval. The hypnosis procedure likely obtains greater control over the subject's inhibition and recall performance. As mentioned earlier, Geiselman, MacKinnon, et al. found that $F$ item recall in the waking state can be predicted significantly from the level of inhibition experienced by subjects under hypnosis. The implication is that some of the mechanisms of forgetting, namely, inhibition processes, are common to both procedures.

The work of Weiner and Reed (1969) suggests that, even when subjects are awake, intent to forget enhances forgetting more than nonrehearsal. Yet recent data from our laboratory have shown that subjects describe their forgetting strategies simply as nonrehearsal. One explanation is that retrieval inhibition requires intent, but, once activated, the inhibition process is carried out passively rather than consciously. Release of the inhibition appears to be more malleable. The present results, for example, indicate that the inhibition can be reversed with a presentation of copy cues of the inhibited items. Current research efforts are being directed toward an examination of the relative effectiveness of alternative classes of suggested active-forgetting strategies and alternative cuing methods for inhibition release. This new research should further our understanding of retrieval inhibition as a process.

One of the practical implications of retrieval inhibition is the more efficient recall of recently presented material. With an active-forgetting mechanism, one can prevent old, out-of-date information from interfering with new, current information. Retrieval inhibition is in some cases preferable to memory erasure in that the old information that is blocked is still available in memory and that information is readily recognizable as having occurred before should it be needed.

\section{REFERENCES}

BJork, R. A. (1970). Positive forgetting: The noninterference of items intentionally forgotten. Journal of Verbal Learning and Verbal Behavior, 9, 255-268.

BJORK, R. A. (1972). Theoretical implications of directed forgetting. In A. W. Melton \& E. Martin (Eds.), Coding processes in human memory. Washington, DC: Winston.

Bjork, R. A., \& Geiselman, R. E. (1978). Constituent processes in the differentiation of items in memory. Journal of Experimental Psychology: Human Learning and Memory, 4, 347-361.

Bugelski, B. R. (1970). Words and things and images. American Psychologist, 25, 1002-1012.

Geiselman, R. E. (1974). Positive forgetting of sentence material. Memory \& Cognition, 2, 677-682.

Geiselman, R. E., BjoRk, R. A., \& Fishman, D. L. (1983). Disrupted retrieval in directed forgetting: A link with posthypnotic amnesia. Journal of Experimental Psychology: General, 112, 58-72.

Geiselman, R. E., Mackinnon, D. P., Fishman, D. L., Jaenicke, C., Larner, B., SchoenberG, S., \& Swartz, S. (1983). Mechanisms of hypnotic and non-hypnotic forgetting. Journal of Experimental Psychology: Learning, Memory, \& Cognition, 9, 626-635.

KinLstrom, J. F. (1977). Models of posthypnotic amnesia. In W. E. Edmonston (Ed.), Conceptual and investigative approaches to hyp nosis and hypnotic phenomena (Vol. 296). New York: New York Academy of Sciences.

Kinlstrom, J. F., \& Evans, F. J. (1976). Recovery of memory after posthypnotic amnesia. Joumal of Abnormal Psychology, 85, 564-569.

REED, H. (1970). Studies of the interference processes in short-term memory. Journal of Experimental Psychology, 84, 452-457.

RoEDiger, H. L., \& Tulving, E. (1979). Exclusion of learned material from recall as a postretrieval operation. Journal of Verbal Learning and Verbal Behavior, 18, 601-615.

WEINER, B. (1968). Motivated forgetting and the study of repression. Journal of Personality, 36, 213-234.

WEINER, B., \& REED, H. (1969). Effects of instructional sets to remember and to forget on short-term retention: Studies of rehearsal control and retrieval inhibition (repression). Journal of Experimental Psychology, 79, 226-232.

(Manuscript received February 1, 1984; revision accepted for publication September 4,1984 .) 